\title{
Self-Orientalization in the East Asian Medical Community
}

\author{
Steve Jackowicz \\ University of Bridgeport, USA, sjackowicz@bridgeport.edu
}

\begin{abstract}
Traditional medical practices have yielded to modern Western Allopathic Medicine in much of the world. However, Traditional East Asian Medicine (aka Traditional Chinese Medicine) which includes Acupuncture, Asian Herbalism, Asian Bodywork, and Meditative Breathing Practices (Taijiquan / Qigong) has grown in popularity around the world. In the United States, Traditional East Asian Medicine has entered mainstream culture, becoming licensed as a type of healthcare service. The development of this type of medicine in the United States began in the late nineteenth century with Asian immigration, but in the latter part of the twentieth century grew from a localized ethnic enclave based cultural practice into a larger social phenomenon reactive to socio cultural dynamics in the medical industrial complex. However, intrinsic in this rise and integration with majority culture has been the inculcation of Orientalized attitudinal poles that rely on stereotypical, trivialized, and racist interpretations of the very traditions that they seem to embrace. This paper examines the aspect of this Orientalizing process in the East Asian Medical community in the United States among immigrants, second generation, and non-Asian students and practitioners.
\end{abstract}

Keywords: Traditional Chinese Medicine, East Asian Medicine, Asian American studies, Medical anthropology, Orientalism

\section{Introduction}

The East Asian Medical community in the US is the byproduct of a number of intersecting social vectors, one of which is a romanticizing Orientalist trend, which mythicizes the remnants of the pre-modern medical system and elevates them to legendary status. This process has been explored previously (Kohn, 2005, Jackowicz, 2009) yet within the American East Asian Medical community there is a self-orientalizing social vector among Asian Americans both immigrants and US born. This paper explores the dynamic of the self-orientalizing process and its impact on the US East Asian Medical community.

\section{Transmission of East Asian Medicine (EAM) to the United States}

In order to contextualize Chinese medicine in the US, a synopsis of the evolution of Chinese medicine is necessary. Chinese medicine has a complex history that I herein separate into four distinct phases; the classical period, the period of Western influence, the republican period, and the communist period (Unschuld, 1985). The classical period spans from the Shang Dynasty through the $18^{\text {th }}$ century. This period is typified by the development of a complex series of correspondences that interrelate the functions of the human physiology with observed and perceived natural phenomena. The medical theory of this long period is consistent in its use of a syllogistic gestalt known as qi (氣). As an axiom of the traditional worldview, the term qi is variously defined and interpreted. Briefly, qi is a coherence of relation that defines a plastic medium of predictable relation. Although translated many times as "energy," that definition falls short of the complexity of the concept in Chinese traditional society (Rose, 2001).

The period of Western influence entails the mid eighteenth through the nineteenth century. Chinese medicine was altered by the changing power dynamic between China and the west. The intellectual colonization of Chinese medicine challenged the axiomatic perception of what is real with a variant worldview and a medicine based on "science." Hybrid concepts of medicine arose with variant levels of success. Further there developed a prejudice against traditional medical concepts as outdated and superstitious (Unschuld, 1990).

The Republican period (1911-1949) influenced Chinese medicine by a wholesale embracing of western medicine as real or correct. Chinese medicine was viewed as a repository of techniques that might have scientific validity. Efforts were made to redeem scientifically amenable aspects of Chinese medicine in a process known as 'scientificization.' In this period hybrid models of traditional techniques with western medical techniques were explored. Further attempts were made to correlate western medical anatomical and 
physiological models with the techniques of Chinese medicine (Chase 1996).

Finally in the Communist Period (1949-present), Chinese medicine has developed into a profession. Altered by the preceding history, Chinese medicine was further changed - revised and redacted by Communist censors to match the material dialectic intrinsic to Maoism. Western medical influence is high in the Communist reorganized Traditional Chinese Medicine (TCM) (Unschuld, 1985).

Additionally, I divide the dissemination of Chinese medicine to the West into four phases; the closed community period, the counter cultural period, the professional licensurate direct pay period, and the professional licensurate third party payer period. The closed community period denotes the time when Chinese medicine was only practiced in the Chinese communities in the West. Beginning with Chinese immigration and developing in the China towns, Chinese medicine in the US was a closed-door phenomenon, which eventually gained a certain level of notoriety among the Caucasian gentility as a niche indulgence. However with no open education system there was little mainstream dissemination of Chinese medical understanding, theory or technique (Culin, 1887).

The counter cultural period in Chinese medicine in the US arose in the 1960's as those disaffected with mainstream culture sought out Chinese medicine as an alternative. This period saw Chinese medical techniques, concepts and approaches being haphazardly co-opted into a 'New Age' approach. The technical training in the US at this time was severely limited, but the interest that developed outside the Chinese community lead to the founding of the first American acupuncture school in 1972, the New England School of Acupuncture (Kaptchuk, 2000).

The counter cultural period ended with the development of professional licenses by the states. Subsequent to the founding of the National Commission for the Certification of Acupuncture and Oriental Medicine in 1982, the states began mandating educational standards and issuing licenses. This change lead to the development of professional schools, which over time were awarded the right to grant master's degrees in Chinese medicine. This period can be identified as the professional licensurate direct pay period since although Chinese medicine in the US was being defined as a profession with specific scope of practice, the insurance system did not embrace it and therefore patients needed to direct pay for services. The development of a restricted scope of practice altered the traditional practices. Also the financial demands of self-pay restricted the population of patients to those with the disposable income who embraced the non-mainstream approach. The financial forces further resulted in an entrepreneurial aspect developing in the Chinese medical community (Kohn, 2005).

The last stage in the history of Chinese medicine in the US is the professional licensurate third party payer period. Although federal worker's compensation covered acupuncture since the late 1970's, beginning in the in the 1990's health insurance programs began to cover Chinese medicine. At first automobile insurance accepted Chinese medicine as post-accident care. However, the watershed in insurance occurred when Harvard Vanguard Health Maintenance Organization was the first to have acupuncturists on staff in their facilities beginning in 1998. Subsequently health insurance carriers have increasingly covered Chinese medicine. Insurance coverage is again altering Chinese medicine since the third party insurance system demands diagnostic coding according to a western medical model of the typification of disease. Chinese medical practitioners must adopt western conceptions of disease in order to be remunerated. This change may perhaps alter the nature of Chinese medicine more than any other.

\section{Variant Views of the "Real"}

Western and Chinese medicine have intrinsically variant views of reality. The Chinese medical model revolves around a worldview wherein a correlative correspondence occurs between the natural world and organic matter. Thus analogy is drawn between natural processes, wind, damp, dry, heat, etc., and metabolic processes. Addressing the imbalances in these natural forces within the metabolism allows the practitioner to normalize the forces and thereby correct health concerns. If the underlying forces are not balanced then health will continue to deteriorate. Therefore, the Chinese medical view of the "real" state of existence is one of complex interactions of forces that are a foundation for visible phenomena (Rose, 2001).

However, Western medicine is based on a different paradigm. In Western medicine, the reductionist view of science is applied to the understanding and typification of disease. Every disease is considered to have one cause that if discovered can be countered. Therefore the Western medical practitioner inspects each system of the body in quantified operation and measures the system for efficiency. If the liver does not match "normal" liver enzyme ranges then the liver is diseased and the liver must be addressed with a medicine that interacts with the 
liver. In Chinese medicine, if the liver is diseased, the systemic interactions of the liver with the other organs of the body, as well as the substrates (qi, blood, nutrition, immunity), or the structure (meridians, bones, muscles), or even the emotions will be inspected and regulated so that the liver returns to normal function (Kohn, 2005). In summary, the Western medical view of the real is that the liver's functions are based in the liver itself, while the Chinese medical view of the real is that the liver's functions only exist in a context of relations. The variant view of reality leads to a variant emphasis in medical approach. Avoiding a discussion of which approach is more accurate, our inquiry will proceed to examine the factors that influence the Asian American practitioners of Chinese Medicine in the US.

\section{Orientalism and Orientalization}

Edward Said advanced the idea of Orientalism in 1979. Said developed an intellectual edifice that advanced the understanding of the psychological dimension of exploitation and colonialism. To summate Said's theory, the West had to reconcile its legendary beliefs in the wisdom of East Asia with the reality of the actual societies they encountered, as well as justify their own exploitative economic colonial devices. Reasoning that east Asia had fallen from once great high culture to the degeneration of the present, allowed the West to exploit East Asia while simultaneously lauding ancient East Asian thought and culture. Said termed this process Orientalism (Said, 1979).

Subsequent scholars have applied this rubric of understanding to examine the Orientalist trends in various fields; historiography, cultural studies, business, etc. Within East Asian Medicine and its transmission to the West, the Orientalist trend has been recognized but not examined in great detail. (Kohn, 2005, Jackowicz 2009). For this inquiry, the process of Orientalism will be considered as the backdrop of the larger socio-cultural process which began in the late 1700's and has persisted in its influence to today. However the nexus of this paper will focus on the specific aspect of Orientalizing the East Asian Medical community in the US and the Self Orientalism and Self Orientalizing process ongoing in the Asian American community.

Orientalizing occurs when a practice, habit, or cultural norm is modified and adapted from its original form to correspond with the Orientalist beliefs that serve as a backdrop to the psychological milieu of the adherents of the practice. Martial arts in the US provide a clear and simple example of Orientalization. A martial art that began in East Asia, when brought to the US, will be re-shaped by its US adherents to conform to the subtle psychological expectations of the adherents. The art may have had very little explicit philosophy in its practice in the place of origin, or it may have had highly cultural specific practices which relate to a religious influence, either of these states will slowly yield to a process of redefinition wherein the art is altered in emphasis and presentation to conform to US social expectations. The consumer driven martial arts market contributes strongly to the forces of redefinition. If the art does not conform to the expectations of the population, it will not draw a base of support. Therefore, in order to survive, the martial art undergoes a transformation within American culture, informed by cinema, stories, and expectations, to become what a martial art is "supposed to be." This process is one of Orientalizing the martial art.

The same process is occurring in the US East Asian Medical community. Although East Asian Medicine, including acupuncture, moxibustion, herbal medicines, therapeutic diet, physical manipulation, massage, and therapeutic breathing exercises, has existed as a medical model for several thousand years, the transplantation of this system to the West has metered it with the backdrop an Orientalist view of the East. Western adherents, as well as patients of the East Asian Medical system in the West, carry a certain baggage of expectation that the medicine of the hoary and learned East will be able to treat them in not only a physical way, but in a wiser method that touches their 'spirit' or 'soul.' These expectations effuse into the schooling delivered in the West in the East Asian Medical field as well as the intellectual edifice of the EAM field in the consumer driven medical arena. To survive, the EAM community perforce adopts and operates within this Orientalist framework. The medical tradition of East Asia is thereby Orientalized to be more akin to a wisdom tradition than a medical practice. The techniques may have medical efficacy, but they become ensconced in a filter of mystical erudition that denies their reality; in effect, they become meaningful beyond their utility.

This process is happening to the community as a whole yet it directly impacts the individuals who comprise the community. Interestingly, only three states in the US allow foreign EAM graduates to gain licensure. The other 45 that license acupuncture require graduation from a US accredited school (Accreditation Commission for Acupuncture and Oriental Medicine, 2018). This requirement is a protectionist element of the states' preservation of homegrown market share. However, it does create a dynamic wherein the Orientalist trend in the US that influences schools and US trained practitioners becomes self-perpetuating. Only New York, 
Massachusetts, and California allow the licensure of foreign-trained graduates. This inquiry will focus on the dynamics in the Asian American community that drives a Self-Orientalizing process, as manifested in immigrant doctors, as well as US trained East Asian Medical providers.

\section{Foreign Immigrant Doctors}

Although EAM has been practiced in the US since the late 1800's with the immigration of the Chinese, post 1972 the rise in interest in the US led to the development of schools and licensure (Culin, 1887, Jackowicz 2009). The rise of a professional licensurate allowed foreign trained providers to become enfranchised in the economics of an emerging medical field and promoted traffic and concourse with the broader mainstream community. Historically this process began in the 1980's and was accelerated by a dissatisfaction with the byzantine insurance edifice and a seeking of alternatives among the mainstream public. These social forces and changing market demands gave full bloom to the growth of acupuncture schools in the US from the first one in 1973 to there now being 46 accredited schools, which outnumber the Western medical schools (Accreditation Commission for Acupuncture and Oriental Medicine, 2018).

However, the foreign-trained provider faced several interactive social vectors. First, the schooling in East Asia (China, Japan, Korea, and Vietnam) for EAM has a decidedly medical bent. The four countries view EAM providers as part of the larger healthcare system and consider them another specialty of medicine (Kohn, 2008). Although there are differences among these four countries' medical systems, their EAM practitioners usually comport themselves like doctors, their education includes a large amount of Western medical information, they have a hospital structure that supports in-patient EAM services, and they are part of the third party healthcare insurance system. Once immigrating to the US, the foreign-trained provider is challenged by a different system. In the US, foreign-trained providers cannot use the title doctor, they enjoy a varied access to insurance billing, there is no in-patient service for EAM, and they have a much more distant relationship to the Western medical establishment (Kohn, 2008). Although there are complex evolutive social vectors in the US EAM field, the above parameters persist and require the foreign-trained provider to enter a commercially competitive marketplace to achieve a market share of patients.

The market demands will influence the foreign-trained provider in several ways. First, the provider may choose only to operate within the ethnic subculture that holds the same value structure as his root culture. Therefore, Chinese trained providers can find a support market in the Chinatowns of New York, Boston, San Francisco, or Los Angeles. These providers may have some mainstream crossover clientele; however, the onus of their practice is made up of the cultural competency group who shares the same understanding and expectations of the type of concern and the type of treatment that will be administered, as well as the expected range of result. Unfortunately, the population of the minority communities is limited in number, locale, and financial resource. Therefore, the foreign-trained provider may choose another option (Yoo, 2012).

The second possibility is that the provider chooses to work within a larger establishment and provide a limited range of services to a pre-selected patient population. For example, a Korean trained provider may find US employment in a clinic that specializes only in back injury. The Korean provider will see patients that have sought the clinic out for back pain relief. The clinic may well have other Western medical providers who case manage the patient's situation and those providers have created an expectation of treatment and potential result in the patient regarding EAM treatment. The foreign-trained provider must then administer treatment but is not bringing to bear the full range of treatment methods, nor does he need to communicate with the patient and manage expectations (Jackowicz, 2017).

The third possibility is that the foreign trained provider will develop a private practice where he attempts to garner as much of the mainstream market share as possible. This situation requires him to interface with the population through advertising, appointment booking, initial intake procedure, defining the scope of treatment, managing expectation, delivering treatment, and managing result. This situation will result in the greatest sociocultural pressure on the foreign-trained provider to alter his behavior pattern. The patient base will not be acculturated to the norms and expectations of the root culture, in fact the exotic fantasy of a "wise" medicine may be driving the patient to find a practitioner. The patient base may be coming with Orientalist expectations of East Asian Medicine. Although the patient may have a serious health concern, he may be seeking just as much emotional solace as physical relief. He may be seeking a "life direction," as opposed to symptom control. The foreign-trained provider is now faced with a dilemma. Either he must change his presentation of himself and start to conform to the expectations of his patients, or he must clearly present himself and potentially alienate the patients (Moran, 2014). 
In a universal healthcare system, such as in East Asia, the provider can rebuke the expectation of the patient and still have a financial resource base of more patients. In the US, the free market forces create a dynamic wherein the provider is hard put to rebuke patient expectations (Wolfe, 2013). Even if the patient is unrealistic to fantastical in his expectations of the "wisdom" or EAM, the provider becomes coopted into a tacit agreement of treating the patient, perhaps with very viable technique, but assuming an identity role that fits the patient's Orientalist expectations (Paik, 2017). The foreign-trained provider is forced to Orientalize himself due to the market forces of private practice, his need to reach a viable patient population, and the substrate assumptions of the US towards East Asia.

\section{US Trained Asian American EAM Providers}

Asian Americans who are either young age immigrants, or US born, undergo a similar process that drives them to Self-Orientalize. Although many Asian Americans are aware of the Orientalist backdrop of US society, and they may deplore it, when attending US EAM training programs they are subtly influenced by the Orientalist themes in the curriculums of accredited schools (Murphy-Shigematsu, 2012).

However, once in practice the US trained Asian American practitioner of EAM faces several choices. Unlike the foreign-trained provider, he has difficulty operating in the culture competency group of his root ethnicity. The Chinese do not take as easily to a US trained provider as to a Chinese trained one. Issues of familiarity, cultural expectation, and xenophobia play into this dynamic. So the Asian American US trained provider is faced with two choices. First, he may seek employment in a clinic, which specializes in one type of concern. The patients attending the clinic are then case managed by the clinic and vetted to see the EAM provider. This option carries the same dynamic for the US trained as well as the foreign-trained provider.

However, the second option is private practice. Akin to the foreign-trained provider, the US trained provider is faced with garnering market share in a free market healthcare industry that does not have universal insurance benefits for EAM. Faced with the dynamic described above, conjoined with an education that carries intrinsically Orientalist themes, the US trained Asian American EAM provider experiences a strong social vector to Self-Orientalize. This may manifest as the acceptance and attribution of treatment results to a more psycho-spiritual or mystical causation, the willingness to treat conditions that have no definable parameters of success (ie. "well-being," "groundedness," "finding my path"), or even subtle erosions of self in the face of acting to a stereotype (faking and accent, emphasizing early birth roots, etc.). The Self Orientalizing process erases some sense of individuality in regards to the societal expectation of "Oriental behavior" (Lee, Erika, 2015).

\section{Vertical and Horizontal Identity}

The dynamic involved in Self-Orientalization correlates to the concepts of vertical and horizontal identity as identified by Andrew Solomon (2013). Vertical identity is an amalgam of the larger genetic, societal, cultural, and historical forces that create a consistent vector of identity, which influences a broad swath of the culture competency group. Horizontal identity is an expression of the personal experiences, attitudes, and interactions that shape a given individual. These two aspects of identity are usually cited as mechanisms to understand and manage cultural differences.

The rubric of identity vectors may be applied to Self-Orientalization. Regarding the immigrant EAM practitioner, the vertical identity is the root cultural conditioning which creates an identity of the practitioner as a "doctor," who regards the tradition as a type of medical science. The coercive social vector of association which drives the practitioner to conform to Orientalist conceptions in the American context creates the horizontal identity of the "wisdom master." The summation of these two vectors on the consciousness of the individual practitioner creates a cognitive dissonance, wherein the imbued aspects of vertical identity are warped and eroded into the horizontal identity. The lateralized translational shift creates a sense of insincerity within the practitioner, which is metered in intensity by personal variation in the original identity matrix (Mooradian, 2006).

Regarding the American trained Asian American practitioner, there is a similar dichotomous identity vector dynamic. However the vertical identity vector is one developed from the American societal structure, which attempts to define an "Asian American-ness" that is distinct from Asian culture (Lee, Jennifer, 2015). The horizontal identity vector encompasses the drive to adhere to the Orientalist fantasy of the American view of 
EAM. The correlation of these two vectors creates a dissonance and a sense of insincerity in the American trained Asian American EAM practitioner.

\section{The Violence of Self-Orientalizing on the Self}

The dissonance between the two aspects of identity induced by the Self-Orientalizing process in either type of practitioner creates an internal violence to the organic sense of self. The need to conform, informed by economic demand, and patient expectation, overwrites the natural sense of self and forces the individual to model behavior patterns, which are scripted externally. The individual is then faced with a loss of integrity and sense of selfcorruption as he acquiesces to presenting himself in a way that is expedient but is not honest. The corruption of self-erodes his ethical base and may provide a venue to the descent into unethical behavior in other venues of his life. Further, this dynamic denies a complete sense of satisfaction and wholeness in the individual, compromising his potential (Benet-Martinez, 2006).

\section{The Violence of Self-Orientalizing on the US EAM Community and Tradition}

The individuals who experience Self-Orientalization suffer personal psychic violence, which corrupts their potential (Lee, Jennifer, 2015). However, this violence creates collateral damage on the community and the tradition. The dissonance creates a sense of subtle shame that poisons the sense of validity in the EAM community, which limits development in the field and gives it an air of charlatanry, which promotes profit over integrity (Wolfe, 2013). Additionally the EAM tradition suffers due to the loss of potential development. The oldest continuously practiced form of medicine is facing a corruption due to social forces rather than medical concerns.

\section{Conclusion}

Self-Orientalization has a subtle yet profound impact on the Asian American practitioners of EAM. The Orientalist platform of American culture coalesces with the vertical and horizontal identity dynamics of the practitioners to create a lateralizing force that degrades the integrity of self, resulting in Self-Orientalization. This process in the Asian American EAM community is one expression of the complexity of the American cultural tapestry wherein dynamics of race and ethnicity combine to either empower or corrupt the population.

\section{References}

The Accreditation Commission of Colleges of Acupuncture and Oriental Medicine Accreditation Handbook (2018). Laurel, MD: Accreditation Commission for Acupuncture and Oriental Medicine.

Benet-Martinez, Veronica \& Shigehiro Oishi. (2006). Culture and Personality. In John, O.P., R.W. Robins \& L.A. Pervin (Eds.), Handbook of Personality: Theory and Research ( pp. 2-52). New York: Gilford Press.

Chase, Charles and Zhang Ting Liang (1997). A Qin Bowei Anthology. Brookline, MA: Paradigm Publications.

Culin, Stewart (1887). The Practice of Medicine by the Chinese in America. Medical and Surgical Reporter: March 19, 1887.

Jackowicz, Steve (2017). An Introduction to Traditional Korean Medicine. International Journal of Chinese Acupuncture. December (17).

Jackowicz, Steve (2009) The Mechanics of Spirit. Berlin, Germany: VDM Publishing.

Kaptchuk, Ted (2000). The Web That Has No Weaver, Second Ed. New York, NY: McGraw Hill.

Kohn, Livia and Steve Jackowicz (2005). Health and Long Life: The Chinese Way. Cambridge, MA: Three Pines Press.

Lee, Erika. (2015). The Making of Asia America. New York, NY. Simon and Schuster.

Lee, Jennifer and Min Zhou (2015). The Asian American Achievement Paradox. New York, NY. Russel Sage Foundation.

Mooradian, Todd A. and K. Scott Swan. (2006). Personality and Culture: The Case of National Extraversion and Word of Mouth. Journal of Business Research, 59, pp. 778-785.

Moran, Robert T. et al. (2014) Managing Cultural Differences $9^{\text {th }}$ ed. New York, NY: Routledge.

Murphy-Shigematsu, Stephen (2012). When Half Is Whole: Multiethnic Asian American Identities. Stanford, CA: Stanford University Press. 
Paik, Susan J, Zaynah Rahman, Stacy M. Kula, L. Erika Saito, and Matthew A. Witenstein (2017). Diverse Asian American Families and Communities: Culture, Structure, and Education. School Community Journal, 2017, Vol. 27, No. 2.

Rose, Ken and Zhang Yuhuan. (2001). A Brief History of Qi. Brookline, MA: Paradigm Publications.

Said, Edward (1979). Orientalism. New York, NY: Vintage Press.

Solomon, Andrew (2013) Far From the Tree: Parents, Children and the Search for Identity. New York, NY. Scribner Press.

Unschuld, Paul (1990). Forgotten Traditions of Ancient Chinese Medicine. Brookline, MA: Paradigm Publications.

Unschuld, Paul (1985). Medicine in China: A History of Ideas. Los Angeles, CA: University of California Press.

Wolfe, Honora and Marilyn Allen (2013). Points for Profit: The Essential Guide to Practice Success for Acupuncturists, New 5th Edition. Torrance: CA: Blue Poppy Enterprises.

Yoo, Grace J, Mai-Nhung Le, Alan Y. Oda (2012). Handbook of Asian American Health. New York, NY: Springer Science \& Business Media. 